\section{IMPROVING STARTING TIME FOR FIRST OPERATING ROOM (OR) CASES IN THE MAIN OR}

Bijad Harbi, Azrul Azam Abdul Aziz, Khadija Alshengite, Emann-Jure S Ascano, Sharifah Bee Ansary, Mariam Abdullah, Azura Abdul Rahman, Ahmad Ali Eid, Kathrina Alam. Surgery Department and Nursing Services, Prince Mohammed Bin Abdulaziz Hospital National Guard Health Affairs

\subsection{6/bmjoq-2019-PSF.69}

Background According to the Safety Reporting System (SRS), there is $54 \%$ compliance for the first OR cases to start on time. An analysis from the OR Benchmarks Collaborative (ORBC) shows the median for the on-time start for first cases is $64.3 \%$. This study was conducted by a multidisciplinary team involving the Surgery Department and Nursing Services. The study includes patients admitted to the surgical unit for an elective procedure inside the main OR. The aim of the study is to increase the compliance rate for the start time of the first OR cases in the main OR from 54\% to $80 \%$ by the end of April 2019, by adhering accurately to the OR start time of $0800 \mathrm{~h}$ for all cases coming from the surgical ward.

Methods A 5-month retrospective study was done by analyzing the generated SRS for the delayed cases. Upon analysis, six reasons were identified. After which, we utilized a Pareto chart to look at the frequency of occurrence for each reason. The outcome measure is the percentage of compliance to the start time of the first case in the main OR at $0800 \mathrm{~h}$. Process measures include the average time needed for preparing patients for surgery in the surgical unit, average time of bringing the patient to the main OR, average time of nursing hand-over and sign-in of the patient in the holding area, completion of consent, and the average time of on-site arrival of surgeons and anesthesiologists. The balancing measure is OR staff average time working hours. A PDSA (plan-do-study-act) cycle was formulated which focused on the pre-operative team and preparing the patient a day prior to the procedure. The PDSA was tested and implemented for a pilot study of 2 weeks and is currently running on its third month of implementation.

Results After implementation of PDSA, the 2-week pilot study achieved a compliance rate of $89 \%$. The compliance rate for February 2019 is at $97 \%$.

Conclusion The project is largely successful due to continuous and effective monitoring. In the future, we seek to organize a Perioperative Committee that will evaluate the patient flow from ward to OR. Furthermore, we aim to expand the project to other units such as Day Surgery and the Endoscopy Unit, and areas that are sending patients to the OR. Ultimately, the overall conclusions are preventing delays of other scheduled cases, improving patient satisfaction, and improving the utilization of OR time.

\section{OPTIMIZING APPROPRIATE DOCUMENTATION OF VENOUS THROMBOEMBOLISM (VTE) RISK STRATIFICATION AND ASSESSING CURRENT VTE PROPHYLAXIS PRESCRIPTION}

Hassan Yousuf, Muhammad K Anis, Jawaher Aljabri, Bashayr Alharbi, M Alfagi, Khadija M Alshengeti. Department of Medicine, Prince Mohammed Bin Abdulaziz Hospital, National Guard Health Affairs

10.1136/bmjoq-2019-PSF.70
Background The aim of the study was to examine the gaps in documentation of VTE risk stratification scoring according to the Padua score in the electronic medical record (BestCare system) for medically admitted patients, and to identify the current practice of VTE prophylaxis according to any guidelines in use.

Methods A concurrent cohort of all patients admitted to the Internal Medicine Department was studied from 1 September to 30 November 2018 for the compliance of VTE prophylaxis documentation and VTE prophylaxis prescription. Data were analyzed in December 2018, a PDSA (plan-do-study-act) model was applied, and educational intervention through presentations and WhatsApp group notifications to the whole medicine team conducted. Monthly data collection was started from January 2019 onwards to identify the improvement after implementation of interventions in our practice. VTE prescription according to National Institute of Health and Care Excellence (NICE) clinical guidelines 2010 was added in BestCare as none existed.

Results A total of 405 patients were studied from 1 September to 30 November 2018. Compliance to Padua score documentation was $94 \%(n=382) .67 \%(n=142)$ patients were at risk of VTE. Among these patients, VTE prophylaxis prescription was not done in 33\%. After implementing interventions in our practice, prospective data collected in January-February 2019 showed significant improvement in complete and appropriate documentation of VTE prophylaxis to $99 \%$ and $98 \%$ VTE prescription according to NICE clinical guidelines 2010. Monthly data will be collected on a regular basis to ensure compliance.

Conclusion The VTE risk stratification documentation and VTE prophylaxis prescription showed promising results after PDSA implementation, introduction of NICE clinical guidelines 2010, and educational interventions. This change has shown an improvement in patient quality of care and safety outcomes.

\section{ADHERENCE TO SCREENING GUIDELINES FOR HEPATITIS AND TUBERCULOSIS AMONG HIV PATIENTS}

Sara AlMunif, Eman AlMazroa, Hanan Balkhy, Majid AlShamrani. Department of Infection Prevention and Control, King Abdulaziz Medical City

\subsection{6/bmjoq-2019-PSF.71}

Background HIV is an infectious disease transmitted through blood or body fluids. The main cause of death for patients with HIV are opportunistic infections (OIs), since patients have a weakened immune system, leaving them susceptible to any infectious agent. OIs are becoming less frequent because of better immunity of HIV patients and earlier screening for OIs for treatment and prevention. As per international guidelines, it is recommended that patients with HIV are screened for hepatitis $\mathrm{B}$, hepatitis $\mathrm{C}$, and tuberculosis upon HIV diagnosis because of their frequent co-infection with HIV and similar route of transmission. However, gaps in screening for common co-infections still occur.

The aim of this study was to estimate the prevalence of hepatitis $\mathrm{B}$, hepatitis $\mathrm{C}$, and tuberculosis among HIV-positive patients, and to assess the adherence of healthcare workers to the screening guidelines of common HIV co-infections.

Methods This was a retrospective analysis of all HIV-positive patients diagnosed at KAMC-Riyadh from 2000 to 2018. Data regarding results of infectious diseases were extracted from each patient's medical file. 